\title{
Expression of vascular endothelial growth factor (VEGF) in IgA nephropathy
}

\author{
Gomathi R. ${ }^{1}$, Adayalam C. ${ }^{2 *}$
}

DOI: https://doi.org/10.17511/jopm.2019.i08.13

\footnotetext{
${ }^{1}$ Gomathi R., Shri Sathya Sai Medical College, Sri Balaji Vidhyapeeth University, Chennai, Tamil Nadu, India.

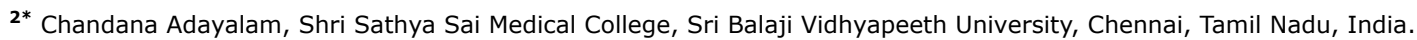

\begin{abstract}
Background: Vascular endothelial growth factor (VEGF) is an important mediator involved in maintaining normal kidney function. Loss of normal or controlled secretion of VEGF following damage to the visceral epithelial cells could lead to alterations in endothelial cell function. This study was carried out to evaluate expression of VEGF in IgA nephropathy in order to look for potential diagnostic markers and predictive markers for progression towards CKD. Methods: This retrospective record based cross sectional study was carried out on a total of 16 whole renal cell biopsy specimens. Following the diagnosis of IgA nephropathy, immunohistochemical techniques were used to evaluate the VEGF expression. The expression of VEGF was graded and evaluated specific for the type of IgA nephropathy. Results: VEGF expression was positive in 6 specimens $(37.5 \%)$. The type of glomerular involvement showing VEGF positivity was mesangial proliferative glomerulonephritis, minimal change disease $(12.5 \%)$, post infectious glomerulonephritis $(6.25 \%)$ and focal-segmental glomerulosclerosis $(6.25 \%)$. All the specimens which showed VEGF positivity were showing well-defined areas with positive cells (25-50\%). Conclusion: Considering the high magnitude of VEGF expression, the present study has potentiated the need for evaluating the statistical significance in attributing VEGF to IgA nephropathy in a larger volume of specimens.
\end{abstract}

Keywords: IgA nephropathy, Immunohistochemistry, Minimal Change disease, Vascular Endothelial growth factor

Corresponding Author

Chandana Adayalam, Shri Sathya Sai Medical College, Sri Balaji Vidhyapeeth University, Chennai, Tamil Nadu, India.

Email: goms318@gmail.com
How to Cite this Article

Gomathi R, Adayalam C. Expression of vascular endothelial growth factor (VEGF) in IgA nephropathy. Trop J Pathol Microbiol. 2019;5(8):585-590. Available From https://pathology.medresearch.in/index.php/jopm/ar ticle/view/307
To Browse

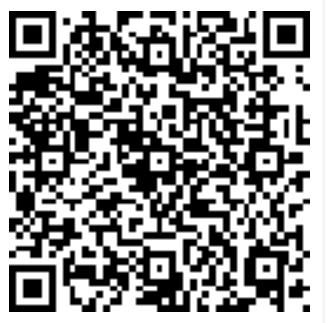

Manuscript Received 2019-07-28

Conflict of Interest No
Review Round 1 2019-08-08

Funding

Nil
Review Round 2

2019-08-16

Ethical Approval

Yes
Review Round 3

Plagiarism X-checker $7 \%$
Accepted 2019-08-22

Note

(C) 2019 by Gomathi R., Chandana Adayalam and Published by Siddharth Health Research and Social Welfare Society. This is an Open Access article licensed under a Creative Commons Attribution 4.0 International License https://creativecommons.org/licenses/by/4.0/ unported [CC BY 4.0]. 


\section{Introduction}

IgA nephropathy is one of the most common causes of chronic kidney disease diagnosed with renal biopsies. Many patients have a slow progressive disease with 30-50\% developing end stage renal disease (ESRD) over a period of 20 years [1]. Histologically IgA is characterized by glomerular and interstitial deposits of IgA in the mesangium accompanied by mesangial hypercellularity and matrix expansion. This mesangial infiltration is believed to be resulting from proliferation of native mesangial cells as well as inflammatory cells, especially cytokines. The development of monoclonal antibodies directed against cell cycle specific antigens has resulted in immunohistochemical methods that allow more precise identification of the proliferating cells in biopsy material [2].

Vascular endothelial growth factor (VEGF) is an important mediator involved in maintaining normal kidney function.It is a potential enhancer of microvascular permeability and a selective endothelial growth factor. In normal human kidney, VEGF $m$-RNA and proteins are strongly expressed by visceral glomerular epithelial cells and VPF/1VEGF may be an important regulator of glomerular endothelial function. In many glomerular diseases, VPF/VEGF expressing cells were decreased in number or absent in areas of focal or global glomerular sclerosis. Similar findings were also observed in amyloidosis, diabetes, crescentic glomerulonephritis, and diffuse endocapillary proliferative glomerulonephritis associated with systemic lupus erythematosus. [3]

Therefore, damage to visceral epithelial cells has a potential for releasing large amounts of VEGF locally resulting in increased glomerular permeability. In addition, loss of normal or controlled secretion of VEGF after the damaged visceral epithelial cells could lead to alterations in endothelial cell function.

An evaluation of VEGF expression in IgA nephropathy has been seldom carried out in countries like India. Although several studies have documented the histological and microcellular involvement of VEGF in renal dysfunction, its potential role in IgA nephropathy has been seldom explored. This study was carried out to evaluate expression of VEGF in IgA nephropathy in order to look for potential diagnostic markers and predictive markers for progression towards CKD.

\section{Objective}

This study was carried out to evaluate the expression of VEGF in IgA nephropathy.

\section{Methodology}

Study setting and participants: This study was carried out in the Department of Pathology in our tertiary teaching institution.

Study duration: This study was carried out for a period of 7 years. A total of 16 whole renal cell biopsy specimens were analysed in this study.

Type of study: This study was carried out as a retrospective record based cross sectional study.

Sampling methods: The specimens for the study were chosen by convenient sampling method.

Sample size calculation: Based on the available literature, the incidence of IgA nephropathy in India varied from $7 \%$ to $16 \%$. [4] At $95 \%$ confidence limits and $12.5 \%$ absolute precision, the sample size was calculated at 15.99 and rounded off to 16 .

\section{Inclusion criteria}

01 . Age above 18 years, both sexes

02. Renal biopsies diagnosed with IgA nephropathy

\section{Exclusion criteria}

01. Absence of IgA nephropathy

02. Pediatric population

Ethical Approval: Approval was obtained from the Institutional Ethics Committee prior to the commencement of the study.

Data Collection: The renal core biopsies were fixed in $10 \%$ formalin and subjected to routine tissue processing. About 4-6 micrometres were cut on a glass slide for routine Haematoxylin and Eosin staining. The diagnosis of IgA nephropathy was made by analysing characteristic histopathological features.

Immunohistochemical staining was done for VEGF by taking up sections in $0.1 \%$ poly-L-lysine coated slides. The sections were labelled using a diamond pencil and placed in slide warmer and 60-degree Centigrade temperature for 30 minutes. It was then transferred to Xylene with 2 changes 5 five minutes each using absolute alcohol and then the slides were gently rinsed in running water. The slides were then placed in $0.01 \mathrm{M}$ citrate buffer with a pH of 6 for pressure cooker heating and the sections were 
Left in the target retrieval buffer at room temperature for 15-20 minutes in the pressure cooker. After boiling, the pressure cooker was cooled by transferring it to a sink in cold running water and the slides were transferred to Tris buffer solution (Tris hydroxyl methyl amino methane). Then, block endogenous peroxidase with $3 \%$ hydrogen peroxidase was used and was rinsed in distilled water for 5 minutes. The slides were then incubated with primary antibody (VEGF) for 1 hour, then further rinsed with Tris buffer solution.

The slides were then incubated with super enhancer followed by rinsing with Tris buffer solution and further incubated with HRP polymer. Later, the excess buffer was wiped and the slides were incubated with substrate/ chromogens for 5 minutes and counterstaining with Hematoxylin was done and was dehydrated and mounted.

Operational definition: The scoring for VEGF expression was as follows (Table 1): [5]

Table-1: Scoring for VEGF expression.

\begin{tabular}{|l|l|l|}
\hline Serial No & Expression & \multicolumn{1}{|c|}{ Interpretation } \\
\hline 1. & - & Negative \\
\hline 2. & + & Few positive cells $(<25 \%)$ \\
\hline 3. & ++ & Well-defined areas with positive cells $(25-50 \%)$ \\
\hline 4. & +++ & Extensive areas with positive cells $(50-75 \%)$ \\
\hline 5. & ++++ & Most cells stained $(>75 \%)$ \\
\hline
\end{tabular}

Data Analysis: Data was entered and analyzed using EpiInfo Version 6.04d. Chi-squared test was used for comparing the VEGF expression among various categories. P-value of $<0.05$ was considered statistically significant.

\section{Results}

This record-based study was carried out among 16 specimens of IgA nephropathy which were evaluated for the expression of VEGF. The majority of the participants belonged to the age group of 2130 years $(37.5 \%)$ and were males $(56.3 \%)$ (Table 2).

Table-2: Background characteristics of the study participants.

\begin{tabular}{|c|c|c|}
\hline Characteristics & Frequency $\mathrm{N}=16$ & Percentage (\%) \\
\hline \multicolumn{3}{|l|}{ Age (in years) } \\
\hline$\leq 20$ & 4 & 25 \\
\hline $21-30$ & 6 & 37.5 \\
\hline $31-40$ & 4 & 25 \\
\hline $41-50$ & 1 & 6.25 \\
\hline $51-60$ & 0 & 0 \\
\hline
\end{tabular}

61-701 6.25 Gender Males 9 56.3 Females 743.7

Table-3: The pattern of glomerular involvement showing Ki67 expression

\begin{tabular}{|l|l|l|}
\hline \multicolumn{1}{|c|}{ Glomerular involvement } & Frequency $\mathbf{N}=\mathbf{1 6}$ & Percentage \\
\hline Chronic tubulo interstitial disease & 1 & 6.25 \\
\hline Mesangial proliferative glomerulonephritis & 1 & 6.25 \\
\hline Crescentic glomerulonephritis & 2 & 12.5 \\
\hline Chronic glomerulonephritis & 1 & 6.25 \\
\hline Post infectious glomerulonephritis & 2 & 12.5 \\
\hline Membranoproliferative glomerulonephritis & 1 & 6.25 \\
\hline Focal segmental glomerulosclerosis & 3 & 18.75 \\
\hline Minimal change disease & 3 & 18.75 \\
\hline Chronic interstitial nephritis & 1 & 6.25 \\
\hline Focal interstitial nephritis & 1 & 6.25 \\
\hline
\end{tabular}

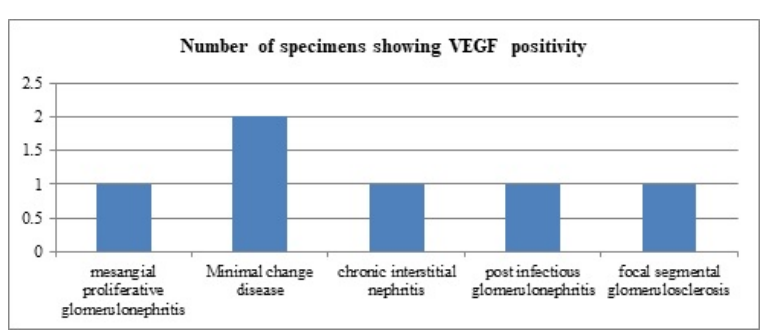

Figure 1: VEGF positivity among the study samples

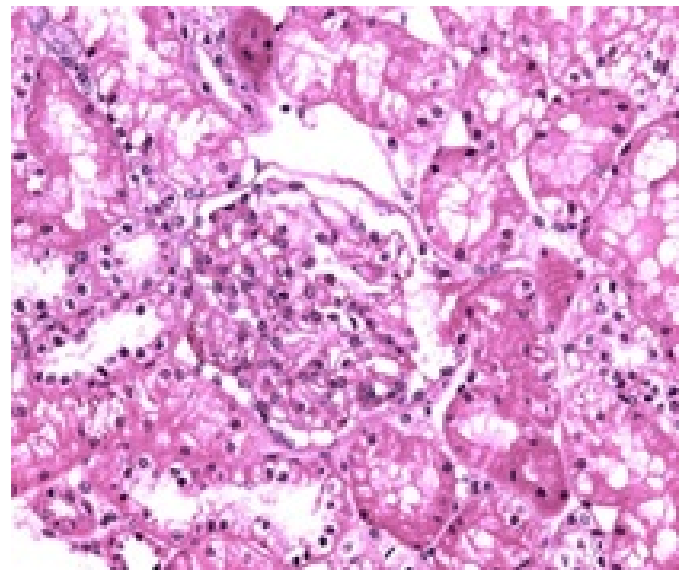

Figure 2: Expression of VEGF in minimal change disease

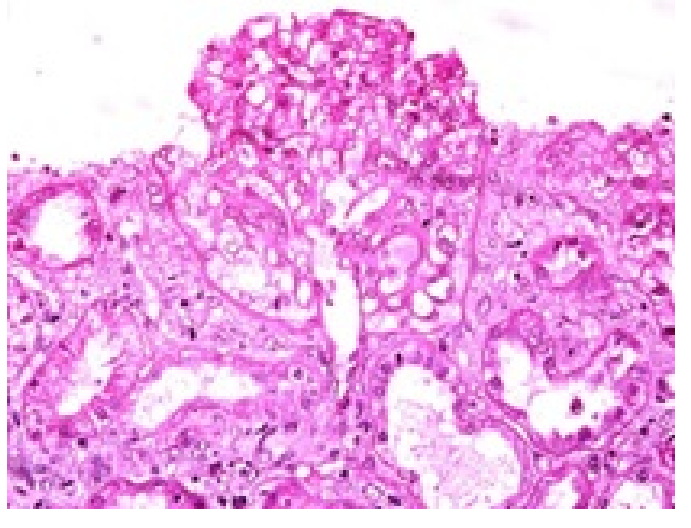




\section{Figure 3: Expression of VEGF in post infectious glomerulonephritis}

The most common pattern of glomerular disease present among the specimens analyzed was focalsegmental glomerulosclerosis and minimal change disease $(18.75 \%$ each). This was followed by post infectious glomerulonephritis and crescentic glomerulonephritis $(12.5 \%$ each) (Table 3$)$. The VEGF expression among the slides studied is given in figure 1. VEGF expression was positive in 6 specimens $(37.5 \%)$. The type of glomerular involvement showing VEGF positivity was mesangial proliferative glomerulonephritis, minimal change disease $(12.5 \%)$, post infectious glomerulonephritis $(6.25 \%)$ and focal-segmental glomerulosclerosis $(6.25 \%)$. All the specimens which showed VEGF positivity were showing well-defined areas with positive cells $(25-50 \%)$. The immunohistochemical profile of VEGF expression is given in Figures 2, 3.

\section{Discussion}

IgAN is potentially implicated in causing slow progressive chronic renal impairment eventually leading on to end stage renal disease. The progression of IgAN is mainly the consequence of arteriolar and interstitial lesions and is found to be independent of IgA deposit magnitude. The renal biopsies of IgAN show a very variable histopathological feature ranging from normal appearing glomeruli to proliferative or sclerosing including extra capillary proliferation. The predominant changes in the glomeruli are mesangial proliferation and focal proliferative nephritis and these are the earliest findings of IgAN [6]. The alteration in the glomeruli tends to be uniform however there could be variation in some cases. The mesangial expansion is better appreciated with PAS stain due to more affinity of mesangial deposits for PAS and the degree of expansion can be determined better using computerized morphometric analysis. Under immunofluorescence, IgA deposits are granular and diffuse in almost all patients however they are occasionally segmental.

Electron dense mesangial deposits that correlate with immune deposits are located in the interphase between the mesangium and the capillary lumina, which is clearly delineated using electron microscopic examination [7]. The prognosis of IgAN is determined by the extent of interstitial fibrosis and tubular atrophy, glomerular sclerosis, capsular adhesions, immune deposits and co-deposition of IgG and IgM and endocapillary proliferation.
Vascular Endothelial Growth Factor (VEGF), is a potential marker for predicting the prognosis of IgAN. VEGF belongs to a family of multipotent cytokines [3]. It is a potential endothelial cell mitogen and is present in the distal tubules and collecting ducts and also in some proximal tubules. VEGF receptors are expressed by endothelial cells of glomerular and peritubular capillaries and mesangium.

They are essentially regulators of angiogenesis. They stimulate endothelial cell proliferation and differen-tiation and also increase vascular permeability and mediates endothelium dependent vasodilatation [3]. The biological effects of VEGF are mediated through tyrosine kinase receptors namely FIt-1 (VEGFR1) and KDR (VEGFR2). They play a major role in early development of vasculature by acting as angiogenic mediator [8].

VEGF expression has been predominantly seen in a wide spectrum of renal diseases, namely glomoreulonephritis of varying forms, nephrotic syndrome and diabetes nephropathy and also in chronic kidney disease (CKD). Their presence has been associated with sepis and atherosclerosis, frequent complications in renal pathologies [9]. VEGF expression has been found to be accelerated early stages of IgAN.

They are also implicated as markers which indicate the recovery of the glomerulus from injury. In the present study, the expression of VEGF in IgAN cases was evaluated and was observed that around $37.5 \%$ of the specimens showed VEGF positivity. The role of VEGF in renal disease has been extensively reviewed by several authors and have shown that activated mesangial cells are source of VEGF especially in proliferative glomerular nephritis.

VEGF also has a role in promoting recovery following glomerular injury, as observed in several published literature [10]. VEGF is said to be upregulated in early stages of renal damage including acute glomerulo-nephritis and early diabetic nephropathy. Exogenous VEGF appears to enhance this process and VEGF has been shown to induce tubular proliferation in metanephric organ culture experiments and has a tubule protective effect [11].

There are very few studies which have explored the role of VEGF expression in IgAN progression. In a study done by Donderski $\mathrm{R}$ et $\mathrm{al}$, there was a statistically significant difference in the VEGF gene 
Expression with various stages of glomerular nephritis including IgA nephropathy, mesangial proliferative nephritis and hypertensive nephropathy. The study also postulated that over expression of VEGF genes may indicate insufficient oxygen supply in the renal tissue thereby resulting in interstitial fibrosis and further deterioration of renal function manifested as impairment in GFR [12]. At the molecular level, inflammatory cytokines including IL10, IL13 may inhibit vascular permeability as a result of which there is over expression of VEGF and resultant proteinuria [3].

However, the protective effect of VEGF on renal tubules is substantiated by stimulation of peritubular microcirculation and peritubular blood flow in addition to peritubular capillary rarefaction. This has therefore resulted in a challenge in the pathognomonic features of VEGF expression in IgA nephropathy. In a study done by Zhai $Y L$ et al, it was observed that patients with IgA nephropathy with severe proteinuria, had higher expression of SFIt-1 receptor of VEGF, thereby indicating that increased expression of VEGF receptors interfered with glomerular epithelial survival, thereby destroying the glomerular filtration barrier, resulting in proteinuria [13].

Although studies have demonstrated the link between expression of VEGF and renal pathology, the causative role of VEGF in IgA nephropathy is largely unclear. Studies have shown that the presence of VEGF expression may be synergistic with the presence of several other factors including glycation end products, plasminogen and plasma glucose levels.

However, the proteinuria in IgA nephropathy can be significantly attributed to VEGF due to chronic upregulation of VEGF in the podocytes, which is essential in maintaining glomerular filtration barrier either directly or through induction of nitric oxide and prostacyclin [14].

Limitation- Since the present study was carried out as a retrospective record-based study, it could not be compared/correlated with the clinical findings and other histological parameters.

\section{Conclusion}

VEGF expression in IgA nephropathy is considerably high in the present study. Expression of VEGF is indicative of endothelium mediated cell proliferation, a predominant feature of hypertensive nephropathy.
Considering the high magnitude of VEGF expression, the present study has potentiated the need for evaluating the statistical significance in attributing VEGF to IgA nephropathy in a larger volume of specimens.

\section{What the study adds to the existing knowledge?}

There are very few studies published in India, on VEGF expression in IgA nephropathy. The present study stands as a pioneer in paving way for further research and hypothesis testing of various receptors of VEGF in our set of population.

\section{Author's contribution}

Dr. Gomathi R. was involved in conceptualization of the study hypothesis and data collection.

Dr. Chandana A. was involved in manuscript writing, editing and review.

\section{Reference}

01. Moriyama T, Tanaka K, Iwasaki C, Oshima Y, Ochi A, Kataoka $\mathrm{H}$, et al. Prognosis in IgA nephropathy: 30-year analysis of 1,012 patients at a single center in Japan. PLoS One. 2014;9(3) :e91756. doi: 10.1371/journal.pone.0091756. [Crossref][PubMed][Google Scholar]

02. Knoppova B, Reily C, Maillard N, Rizk DV, Moldoveanu Z, Mestecky J, et al. The Origin and Activities of IgA1-Containing Immune Complexes in IgA Nephropathy. Front Immunol. 2016;7:117. doi: 10.3389/fimmu.2016.00117.eCollection2016 [Crossref][PubMed][Google Scholar]

03. Schrijvers BF, Flyvbjerg A, De Vriese AS. The role of vascular endothelial growth factor (VEGF) in renal pathophysiology. Kidney Int. 2004;65(6)20 03-17. doi: [Article][Crossref][PubMed][Google Scholar]

04. Chacko B. IgA nephropathy in India: what we do know. Ren Fail. 2011;33(1):102-107. doi: 10.3109/ 0886022X.2010.523486 [Crossref][PubMed][Google Scholar]

05. Namikoshi $T$, Satoh $M$, Horike $H$, Fujimoto $S$, Arakawa S, Sasaki T, et al. Implication of peritubular capillary loss and altered expression of vascular endothelial growth factor in IgA nephropathy. Nephron Physiol. 2006;102(1):p9-16. doi: 10.1159/ 000088405 [Crossref][PubMed][Google Scholar] 
06. Alsaad KO, Herzenberg AM. Distinguishing diabetic nephropathy from other causes of glomerulosclerosis: an update. J Clin Pathol. 2007;60(1):18-26. doi:10. 1136/ jcp.2005.035592 [Crossref][PubMed][Google Scholar]

07. Wallace $E$, Maillard $N$, Ueda $H$, Hall S, Fatima $H$, Novak J, Julian BA. Immune profile of IgA-dominant diffuse proliferative glomerulonephritis. Clinic Kid J. 2014; 7(5):479-483. doi: 10.1093/ckj/sfu090 [Crossref][PubMed][Google Scholar]

08. Ferrara N. Role of vascular endothelial growth factor in regulation of physiological angiogenesis. Am J Physiol Cell Physiol. 2001;280(6):C1358C1366. doi: 10. 1152/ajpcell.2001.280.6.C1358 [Crossref][PubMed][Google Scholar]

09. Lim AKh. Diabetic nephropathy - complications and treatment. Int J Nephrol Renovasc Dis. 2014;7:361-81. doi: 10.2147/IJNRD.S40172. eCollection $2014 \quad$ [Crossref][PubMed][Google Scholar]

10. Doi K, Noiri E, Fujita T. Role of vascular endothelial growth factor in kidney disease. Curr Vasc Pharmacol. 2010; 8(1):122-128. doi : $10.2174 / 1570161 \quad 10 \quad 790226606$ [Crossref] [PubMed][Google Scholar]
11. Kim BS, Goligorsky MS. Role of VEGF in kidney development, microvascular maintenance and patho-physiology of renal disease. Korean J Intern Med. 2003;18(2):65-75. doi:10.3904/kjim.2003.18.2.65 [Crossref][PubMed] [Google Scholar]

12. Donderski R, Szczepanek J, Domagalski K, Tretyn A, Korenkiewicz J, Marszałek A. Analysis of relative expression level of VEGF (vascular endothelial growth factor), HIF-1a (hypoxia inducible factor 1a) and CTGF (connective tissue growth factor) genes in chronic glomerulonephritis (CGN) patients. Kidney Blood Press Res. 2013;38(1):83-91. doi: 10.1159/00035 5754. Epub 2014 Feb 22 [Crossref][PubMed][Google Scholar]

13. Zhai $Y L$, Zhu L, Shi SF, Liu LJ, Lv JC, Zhang $H$. Elevated soluble VEGF receptor sFlt-1 correlates with endothelial injury in IgA nephropathy. PLoS One. 2014;9(7):e101779. doi:10.1371/journal.pone. 0101779. eCollection 2014 [Crossref][PubMed][Google Scholar]

14. Liu E, Morimoto M, Kitajima S, Koike T, Yu Y, Shiiki $H$, Nagata $M$, et al. Increased expression of vascular endothelial growth factor in kidney leads to progressive impairment of glomerular functions. J Am Soc Nephrol. 2007;18(7):2094-2104. Epub 2007 Jun 6. doi:10.1681/ASN.2006010075 [Crossref][PubMed][Google Scholar] 